\title{
Onychomycosis by Paecilomyces lilacinus in a patient with leukonychia
}

\author{
Patrizia Innocenti', Elisabetta Pagani', Dunja Vigl', Valentina Pasquetto', Katia Scalzo', Clara Larcher' \\ I Laboratorio Aziendale di Microbiologia e Virologia, Comprensorio Sanitario di Bolzano \\ 2 Reparto di Dermatologia, Comprensorio Sanitario di Bolzano
}

Key words: Paecilomyces lilacinus, ITS, PCR, cycle sequencing

\section{Onicomicosi da Paecilomyces lilacinus in paziente con leuconichia}

\section{SUMMARY}

Paecilomyces lilacinus, a filamentous fungus living saprophytic in the environment, very rarely causes infection in immunocompetent humans, mostly ocular or cutaneous and sub-cutaneous infections. The fungal isolates usually show low susceptibility to conventional antifungal drugs in vitro and variable susceptibility to novel triazoles.

During a period of 2 years, 4 cultures of nail fragments were grown from a $4 \mathrm{I}$ years old female on modified Sabouraud/Dextrose agar (S.D.A.) (CAF bioMérieux, CAF+CEX Biolife). Cultures were continued in suspension and the hyphae stained with lactophenole blue analysed microscopically. 2 samples were tested with molecular methods, i.e. DNA extraction, amplification of internal transcribed spacer (ITS) region $I$ and 2 and automated sequencing. The sequence results were evaluated using a validated reference database for humanpathogenic fungi. Additionally the BLAST algorithm on NIH database GenBank was used.

A growth of a filamentous hyaline fungus with white colonies and lila shade was observed in all samples. The presence of Penicillium like conidiophores with divergent long terminal phialides was seen with little elliptical conidia. DNA sequencing determined the species as Paecilomyces lilacinus with $99 \%$ homology with GenBank isolate AY2I 3667.

The infection of the right hallux did not improve by systemic (Lamisil/terbinafine) and local treatment (Locetar/amorolfin nail lacquer). Also change to Sporanox/itraconazol only led to deterioriation, so finally the extraction of the nail had to be performed given the known difficulties with treatment described in the literature.

This case highlights the usefulness of molecular techniques, especially use of the highly discriminative ITS region for fungal typing.

La diagnostica micologica si basa a tutt’oggi, in particolare per i miceti filamentosi, principalmente sull'identificazione fenotipica, ma il ricorso alle tecniche di biologia molecolare si rivela talora di grande aiuto, soprattutto quando il microrganismo sia di raro riscontro o di difficile identificazione con i soli caratteri fenotipici.

A tale proposito, descriviamo di seguito il caso di una paziente affetta da leuconichia, in cui ripetuti isolamenti colturali, confermati dall'analisi di sequenza, hanno evidenziato la presenza di Paecilomyces lilacinus, micete ialino, saprofita ambientale di raro riscontro quale agente causale d'infezioni in soggetti immunocompetenti, solo occasionalmente descritto come agente d'infezione alle unghie (3).

Da novembre 2006 a ottobre 2008 sono state allestite 4 colture da frammenti d'unghia, prelevati dall'alluce sinistro di un soggetto immunocompetente (41 anni, sesso femminile) che si è presentato al Reparto di Dermatologia del Comprensorio Sanitario di Bolzano con un ispessimento e alterazione del colore della lamina ungueale riconducibili a distrofia e leuconichia. La paziente, inizialmente sottoposta a terapia antimicotica sistemica (Lamisil/Terbinafina 250 $\mathrm{mg} /$ die) e locale (Locetar/Amorolfina lacca ungueale) per oltre quattro mesi non ha dimostrato alcun miglioramento clinico ed è stata, quindi, successivamente trattata per via sistemica con Sporanox/Itraconazolo (100 mg), mantenendo invariata la terapia locale. I valori dei markers per la valutazione della funzionalità epatica, misurati in questo periodo, risultavano nella norma. Ad un leggero miglioramento iniziale dopo il cambio terapia è seguito un peggioramento del quadro clinico con interessamento dei due terzi dell'unghia; si è deciso, pertanto, per l'estrazione dell'unghia, eseguita ambulatorialmente in anestesia tronculare.

I frammenti d'unghia sono stati posti in coltura su Agar di Sabouraud modificato (S.D.A.+CAF bioMérieux Italia) e Agar selettivo per dermatofiti (S.D.A. CAF+CEX Biolife), incubati fino a tre settimane a $30^{\circ} \mathrm{C}$ in aerobiosi; dopo la prima evidenza di crescita è stata allestita una coltura su vetrino al fine di osservare al meglio le strutture fungine e l'analisi microscopica è avvenuta con ingrandimento $100 \mathrm{X}$ e 400X.

Per due dei quattro isolati é stato affiancato il sequenziamento genico delle regioni ITS (Internal Transcibed Spacer) 1 e 2. L'estrazione degli acidi nucleici è avvenuta utilizzando il sistema automatizzato NucliSENS ${ }^{\circledR}$ easyMAG TM (bioMérieux), previo shock termico e meccanico della sospensione fungina. $5 \mu \mathrm{l}$ di DNA estratto sono poi stati utilizzati per la sintesi degli ampliconi, adattando i protocolli descritti da Chen C, et al $(1,2)$. I primers e le condizioni applicate sono riportati nella figura I. I prodotti di amplificazione, visualizzati mediante corsa elettroforetica su gel d'agarosio, sono stati sequenziati utilizzando il prodotto BigDye Terminator v 3.1 RR-cycle sequencing kit, associato allo strumento ABI Prism 3130 Genetic Analyzer (Applied Biosystems, Foster City, CA), previa purificazione dei prodotti di PCR (ExoSAP-IT, GEhealtcare, Milano, Italia).

Le sequenze ottenute sono state quindi allineate con il database appositamente creato mediante il sistema TraceEdit Pro, RidomBioinformatics, in seguito ad un'approfondita ricerca bibliografica relativa alle specie funginee patogene per l'uomo (4; sequenze di riferimento raccolte da Blast/GenBank http://www.ncbi.nlm.nih.gov/blast/Blast.cgi) e grazie ai preziosi suggerimenti del Prof. Hartwig P. Huemer (Department of Hygiene, Microbiology and Social Medicine, Innsbruck Medical University, Innsbruck, Austria). Al fine di non precludere la possibilità d'individuare specie meno conosciute in ambito patologico per la specie umana in aggiunta è stato consultato il sito http://www.ncbi.nlm.nih.gov/blast/Blast.cgi L'esame colturale dei quattro diversi prelievi ungueali ha evidenziato la crescita su S.D.A. + CAF di un micete ialino filamentoso, con colonie sviluppatesi in un periodo di tempo di 4-5 giorni, dall' aspetto vellutato, di colore bianco con sfumature tendenti al lilla e retro della colonia pallido.

Inoltre, l'analisi microscopica e la coltura su vetrino hanno permesso di evidenziare la presenza di conidiofori Penicillium-like con fialidi terminali molto allungate a fiasca, con piccoli conidi lisci ovoidali/elissoidali. Tali caratteristi-

\section{Corresponding author: Patrizia Innocenti}

Laboratorio Aziendale di Microbiologia e Virologia

39100 Bolzano - Comprensorio Sanitario di Bolzano - Via Amba Alagi 5 - Tel. 047I-909627 - Fax 047I-27263।

E-mail: patrizia.innocenti@asbz.it 
che morfologiche hanno permesso di ricondurre la specie coinvolta a Paecilomyces lilacinus.

Gli allineamenti relativi ai consensus, ottenuti previo assemblaggio delle sequenze condotte nelle due direzioni (forward e reverse), hanno confermato in maniera inequivocabile il risultato dell'indagine microscopica in entrambi gli isolati per cui è stata eseguita l'analisi di sequenza; è stata dimostrata infatti, relativamente alla regione genomica analizzata, un'omologia del 99\% del materiale genetico del micete isolato nel nostro Laboratorio con la specie Paecilomyces lilacinus (accession number GO229080, AY213667, AB244777). Il risultato è stato inoltre confermato dall'analisi condotta confrontando le sequenze ottenute con la banca dati creata mediante il sistema Ridom/TraceEdit Pro.

I risultati ottenuti dai diversi isolamenti confermano in primo luogo Paecilomyces lilacinus quale occasionale agente eziologico di onicomicosi. Essi ribadiscono inoltre, in particolare per i miceti filamentosi, il ruolo dell’identificazione fenotipica nella diagnostica micologica. Le evidenze ottenute sottolineano altresì che il ricorso alle tecniche di tipizzazione molecolare altamente specialistiche, quale il sequenziamento genico, si rivela di grande aiuto quando il microrganismo sia di raro riscontro o di difficile identificazione con i soli caratteri fenotipici. Infatti, benché le caratteristiche morfologiche indicassero Paecilomyces lilacinus quale micete coinvolto, la quasi completa mancanza in letteratura di casi di onicomicosi in paziente immunocompetente ci ha indotto a confermare l'identificazione del fungo con la caratterizzazione molecolare, completando in maniera esaustiva l'analisi del micete evidenziato nella patologia osservata.

\begin{tabular}{lccc} 
REGIONE & NOME & SEQUENZA & DIMENSIONI \\
\hline ITS & ITSI (forward) & $5^{\prime}$-TCCGTAGGTGAACCTGCGG-3' & $500-600$ bP \\
\hline & ITS4 (reverse) & $5^{\prime}-$ TCCTCCGCTTATTGATATGC-3' & \\
\hline $\mathbf{N}$. cicli & & & estensione \\
\hline I & denaturazione & ibridazione & $72^{\circ} \mathrm{C} \times 30^{\prime \prime}$ \\
\hline 28 & $95^{\circ} \mathrm{C} \times 5^{\prime}$ & $55^{\circ} \mathrm{C} \times 30^{\prime \prime}$ & $72^{\circ} \mathrm{C} \times 30^{\prime \prime}$ \\
\hline l & $95^{\circ} \mathrm{C} \times 30^{\prime \prime}$ & $55^{\circ} \mathrm{C} \times 30^{\prime \prime}$ & $72^{\circ} \mathrm{C} \times 7^{\prime}$ \\
\hline
\end{tabular}

Figura I. Sequenza dei primers utilizzati nell'identificazione molecolare dei miceti a livello della regione ITSI-2 e condizioni di amplificazione applicate

\section{RINGRAZIAMENTI}

Gli Autori desiderano ringraziare il Prof. Hartwig P. Huemer (Department of Hygiene, Microbiology and Social Medicine, Innsbruck Medical University, Innsbruck, Austria) per il prezioso aiuto.

\section{BIBLIOGRAFIA}

1. Gurierrez-Rodero F, Moragon M, Ortiz de la Tabla V, Mayol MJ, Martin C. Cutaneous hyalohyphomycosis caused by Paecilomyces lilacinus in an immunocompetent host successfully treated with itraconazole: case report and review. Eur J Clin Microbiol Infect Dis 1999; 18 (11): 814-8.

2. Chen YC, Eisner JD, Kattar MM, et al. Identification of Medically Important Yeasts Using PCR-Based Detection of DNA Sequence Polymorphisms in the Internal Transcribed Spacer 2 Region of the rRNA Genes. J Clin Microbiol 2000; 38: 2302-10.

3. Chen YC, Eisner JD, Kattar MM, et al. Polymorphic Internal Transcribed Spacer Region 1 DNA Sequences Identify Medically Important Yeasts and Brad T. Cookson J Clin Microbiol 2001; 39: 4042-51.

4. Rakeman JL, Bui U, Lafe K, Chen YC, Honeycutt RJ, Cookson BT. Multilocus DNA sequence comparisons rapidly identify pathogenic molds. J Clin Microbiol 2005; 43: 3324-33. 